\title{
qhsuluduh USחกntUltr
}

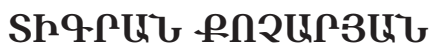

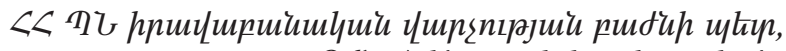

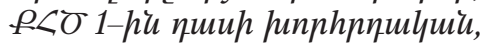

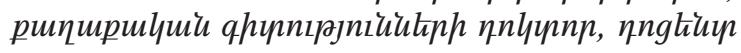

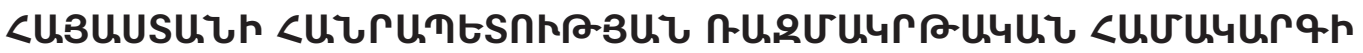

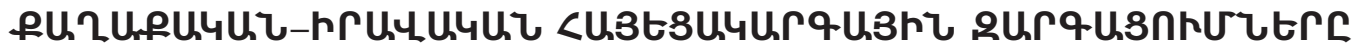

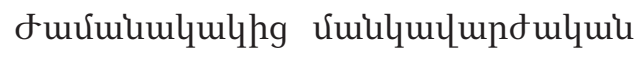

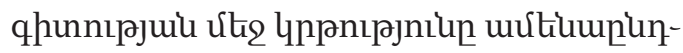
huinıp ununkgưứp utiplqujuglnuर

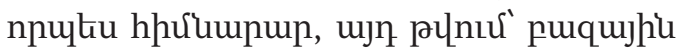
umulumqhumuluiu qhonkhputiph thn-

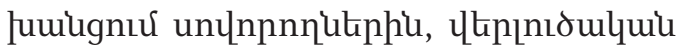

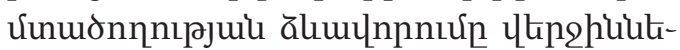

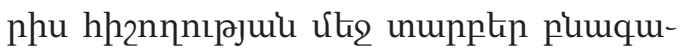

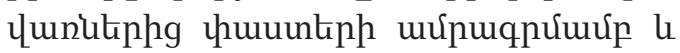

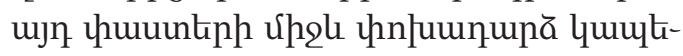
nh nnnzwly hưumlqunqh ăumunnưuŕp,

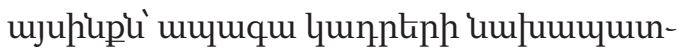
nuuunnuxp uihhuju hnulh6ulqutennıx

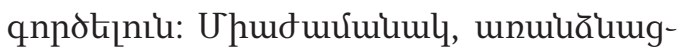

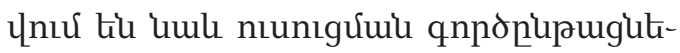

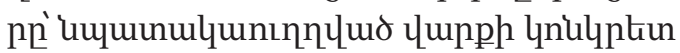

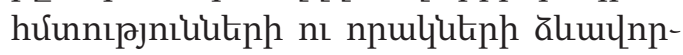
umun, uju 5 ' umwqu gunptiph 'umpumưuunnuuunnıúp npnzwlhnptiu hujunup

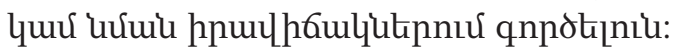

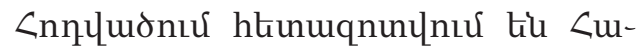

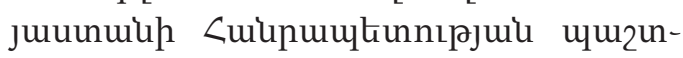

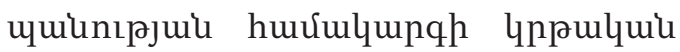
qunnıguluunqtiph qujugưuu nınhu, uqqujhu lnpaulquiu thnnāh lu nuqưulnpulquil nцnpunnux uknh nitignn ưh-

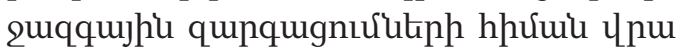
hpulquiumgцnn hujkgulqunqujhi punk-

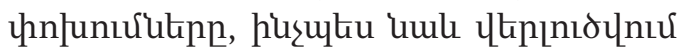

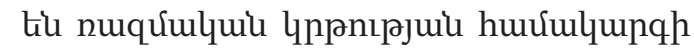

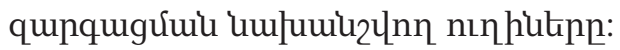

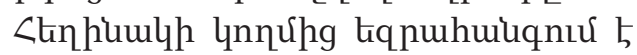

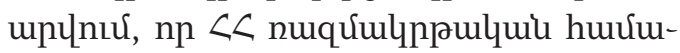

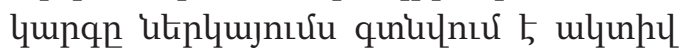

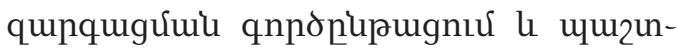

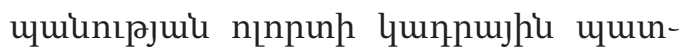

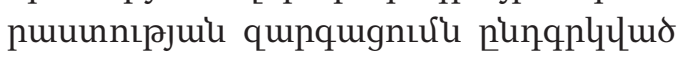

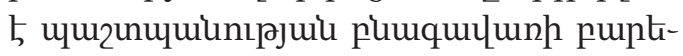

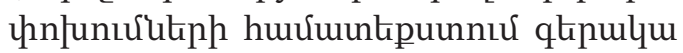

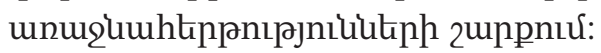

<uiuqnigujhì punten lo mprnwhwy-

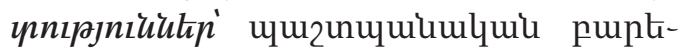
unpunıưute, nuqưuluiu दnpnıpjniu, hujtggulump, qhüounujnn, nuqưunt-

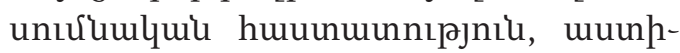

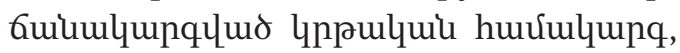
nuqưulquil qhunnıpjnil, úmulumqhunuquil uquinnuuunnıрjnıl:

Gtplymjnıরu Cujuunmilh Cuipumk-

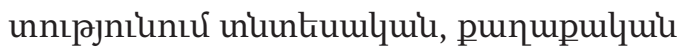

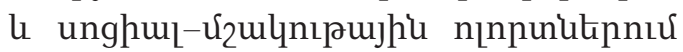

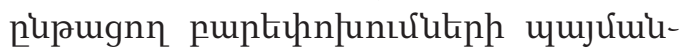

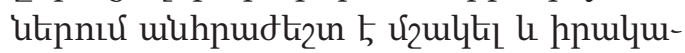

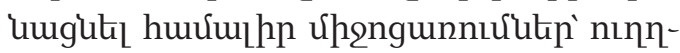

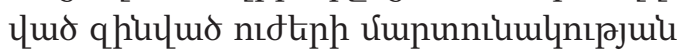

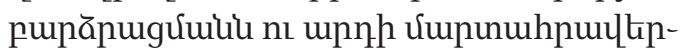

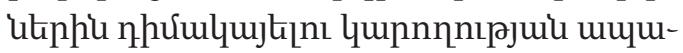

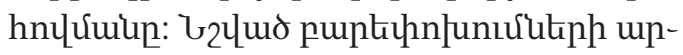




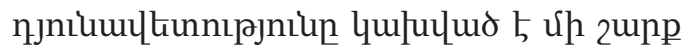

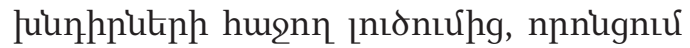

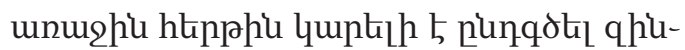
цuљ nıdtph qunnıgluopulqunnujhu

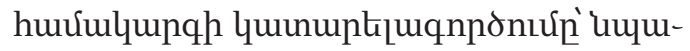

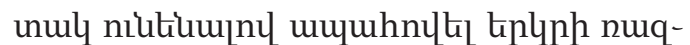

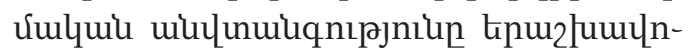
nnn puiuul [1]:

burulhi bunphpnujhi ujnıu huilpuukinnıpjniukteph htiun qptipt úpudưưuml, umluju unulth mupuptiumuun

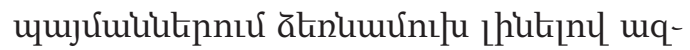
qujhi puiumlh uuntindưuln, <ujuuunu-

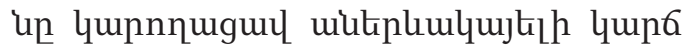

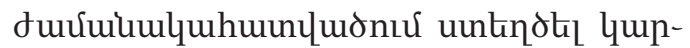
quuwh nı úmunniuml qhuluo nıdtip, npnip utiplujnıư hpting umpunuluiu

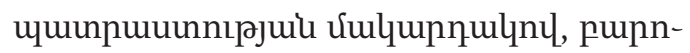

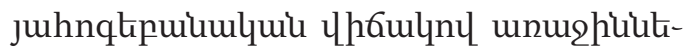
nhg tiu UT<-nuर, hul <upullynllyuujui

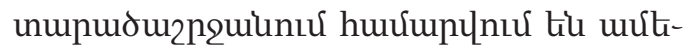

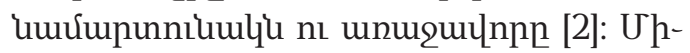

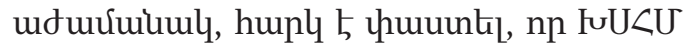

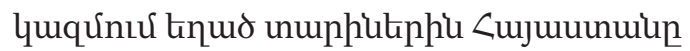

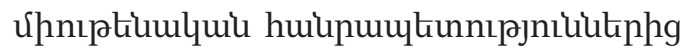
úpuliu $5 n$, nph unupuopnư slump npls, nuquulpnpulqui huunwunnıpjnil: furgh ujn, umpulhi foU $\angle U$ puiumlh ump-

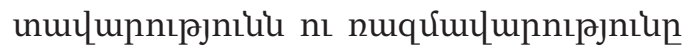

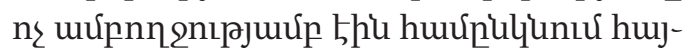

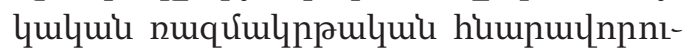
pjniuutiphu:

1994 plulumup hniuhupg hkinn piu-

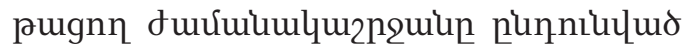

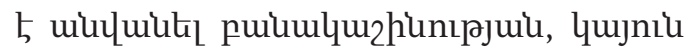
qunqugưuil, qnpptiph ưununniumlnnpjuiu punānugưuil, qunquumuhnıрjui

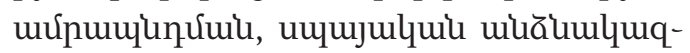

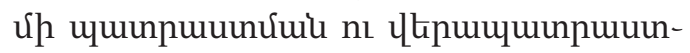
umu, husuku umb puimul-huumpu-

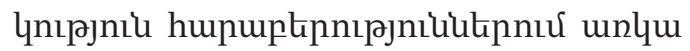
mignukinh ununkgununh lthnugưuil $2 \mathrm{n}^{-}$

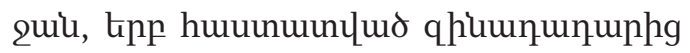

htiun tiplph h2luwunıpjniuutenn huw-

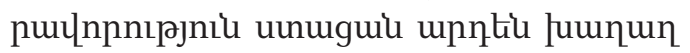

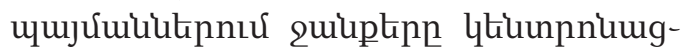

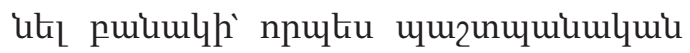

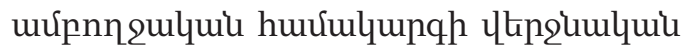

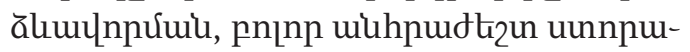

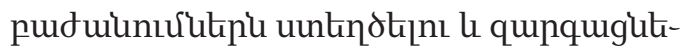
Łnı nıпппцрјшưр:

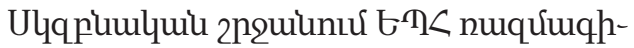

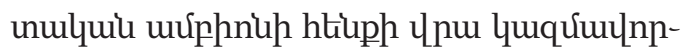

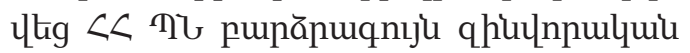

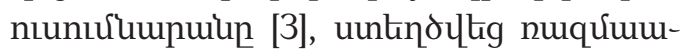

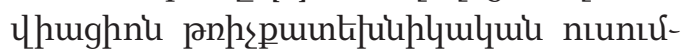

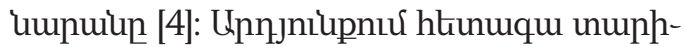

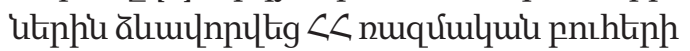
hưumlympn, umukmunnmulku, $\angle C$ TT pupănuqnuju qhulnpulquiu nıunıúum-

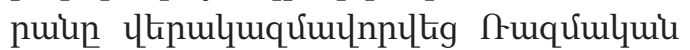
huuunhunnunh [5], hul nuqưumulhugh-

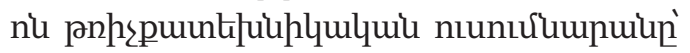
ก-uqưumulhughnu huuunhunnunh:

¿< qhuyur nıdtiph nuqưupd 24 u-

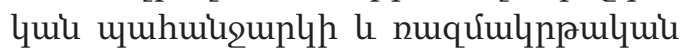
unuiuăuwhuunlnıpjntuktenh hu2lun-

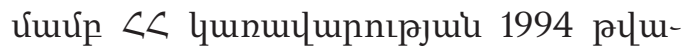
quiuh umjhup 19-h phl 232 nnnzưứp

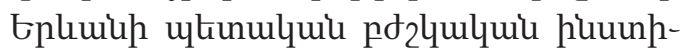
unnıun pnıdðunujnıрjuil quqưultinu-

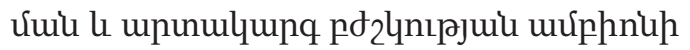

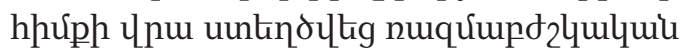
\$ulynıluntinn [6]: <ứuăuju $<<$ yunu-

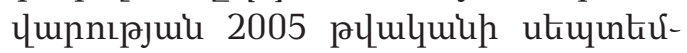

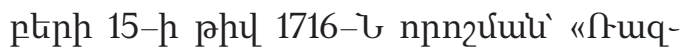

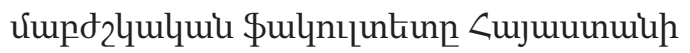

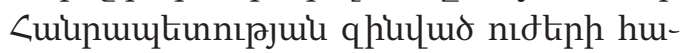
ump umunpumunnuर 5 nuqưupd 2 quilumu pupănuqnıju цnpnıpjứp uщuiten, quqưulthnunıu 5 htionnnıhulquiu la htiu-

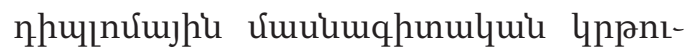

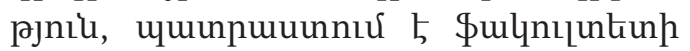

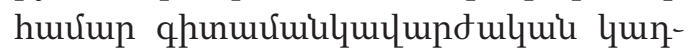

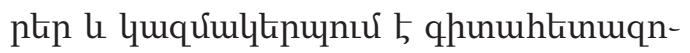
unulquiu u2lumunuiphikp»" [7]: Uju pn[nph 


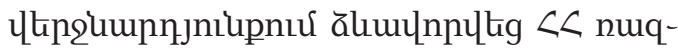

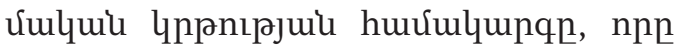

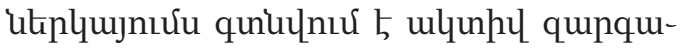

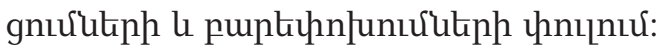

ก-uqưunltiunh, nuqúulquil qhunn-

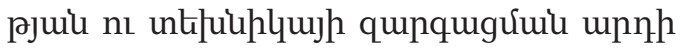

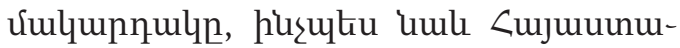

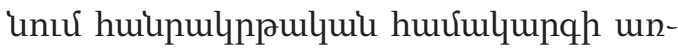

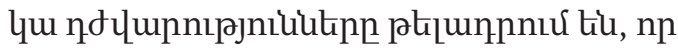

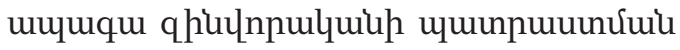
qnpopupugnuर ukinp 5 2

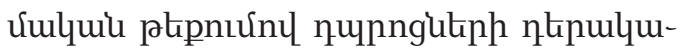
ununnıрjniun: Ujn huuunuunnıрniuutiph

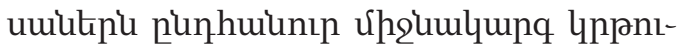

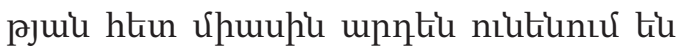

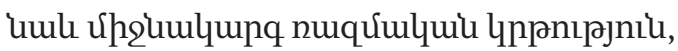
\$hqhlumula, unuunnumbu ns hnqtipuunptiu umunpuunulur tiu htionuqu nıuumil lu dunujnıрjuil huर्ump: Ujn 2nqu-

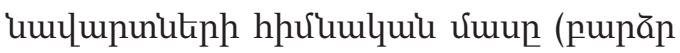
unųunhưnıpjnılu gnıguptennnutinn) uhuiuquúng lnpnıpjnilu zunniluwht-

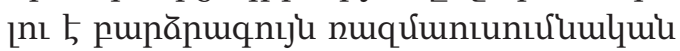

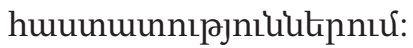

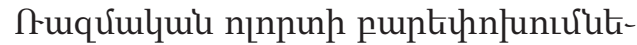

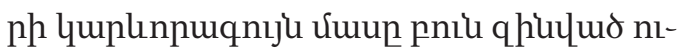

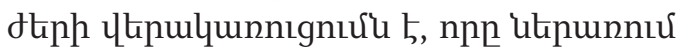

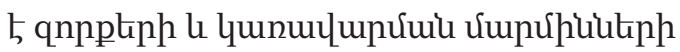

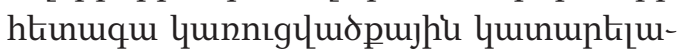
qnnonıúp, qhulud nıdtph úmpnulyui

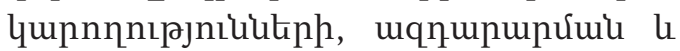
qunuцunúu hưumlymph ouknump-

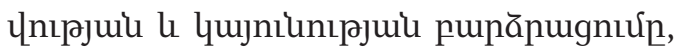

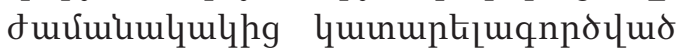
qhimunkumlitipnl puiumlh uщunuqhunıрjuiu hưưunnıúp: 3nıрupwizjnıр ukinnıpjniu hn nuqưulquiu wulunuiqqnt-

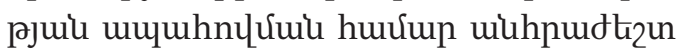

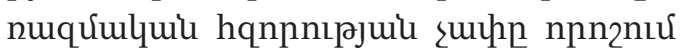

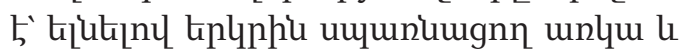
humpuцnn цunuiqh duцujuknhg, hp unu-

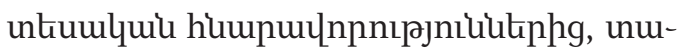

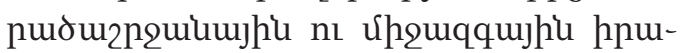

lhfoulhg, nıdtiph unupudmanguiumjhi

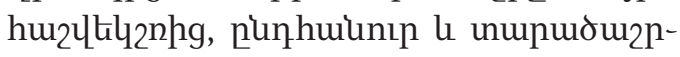

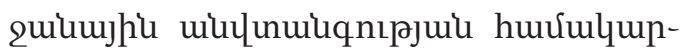
qnư hp unuiuăumd umpunuцnnnıрjniu-

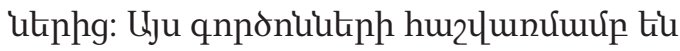

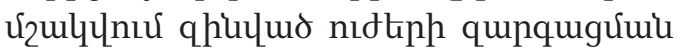
nupughl lu htnuilqunujhi dnuqptinn,

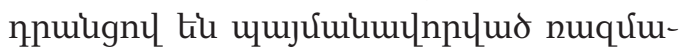
quiu 2humpunnıpjuiu zwun hungthn, ujn-

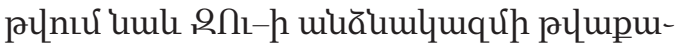

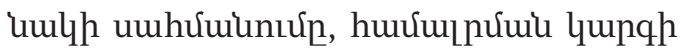
nnn२nuú:

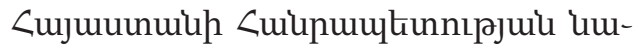
luwquhh lnnर्uhg 2007 plulquilh hnilh-

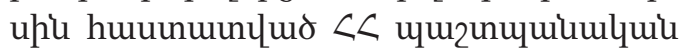
pupkihnjunıưlikp 2007-2015 plulquiu-

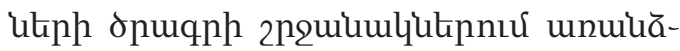
uwlh nızunpnıpjnilu whinp 5 nupăuks uml qhiulud nıdtpnou qhiunnulumi

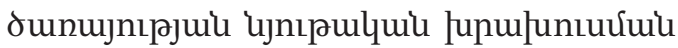

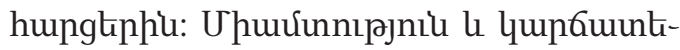

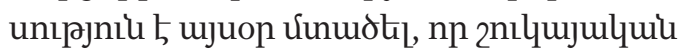

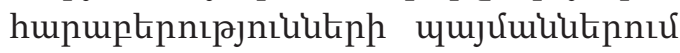

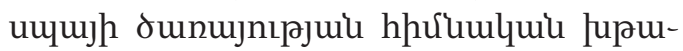
upsn uhuju dunujnпulqui jumunuцunnıpjnilu 5 nı hujntiumupnulquiu ưnn-

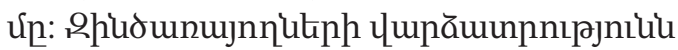
ujuop ujupwi $5 \mathrm{l}$ ch huर्umumunuulum-

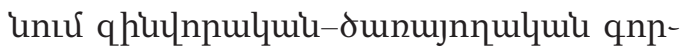
dunnıplkiph pupnnıрjuin, 4uplennnı-

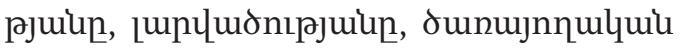
umunumlquinıpjniuitiph huinnil punts-

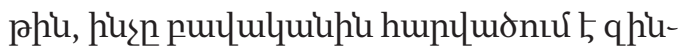

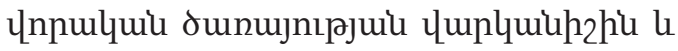
nрulyjul quinptipnl qhiluo nıdtiph huumunuiu qnpopupughi: शhudunujnnh

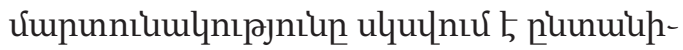

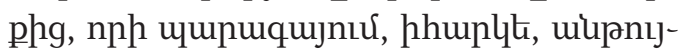
juinptilh 5 qhidounujnn hiu unghu[ulquil

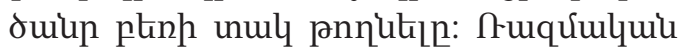

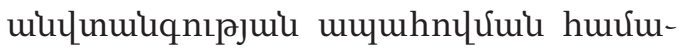
qupqnuर uipnıjuinptilh 5 unghu[ulquil

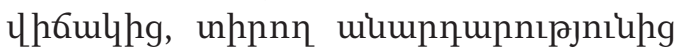




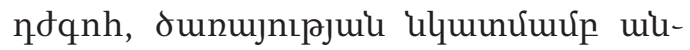

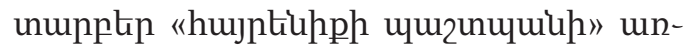
цujnıрjniun:

Luj uuwui ukinp 5 hhip uqqujhu

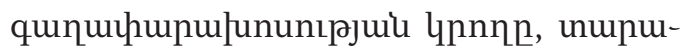

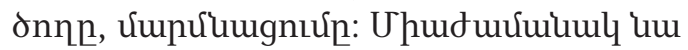
ukinp 5 huuwpulnnıpuiu lnnर्uhg piu-

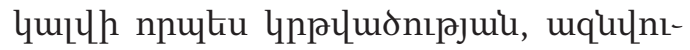

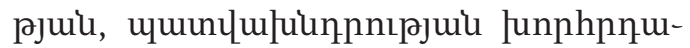

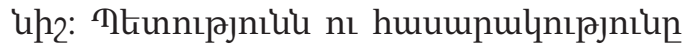

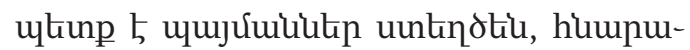

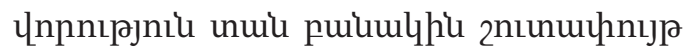
wquinцtil pn_np upuighg, nlptep puiuml

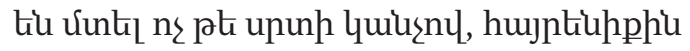

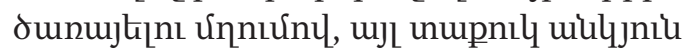

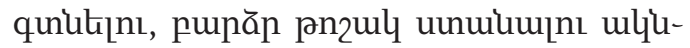

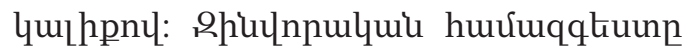
unjuuktu ukinulquiu npnzhu hưưndtip

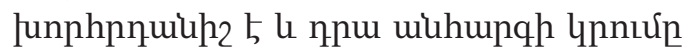
huluuupuqne 5 huiuguqnnonıpjuiu

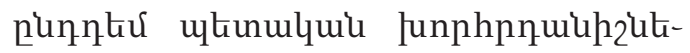

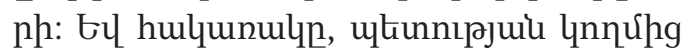
qhuцnnulquiuteph punăn quwhumnt-

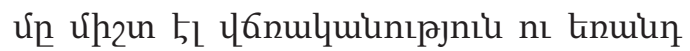

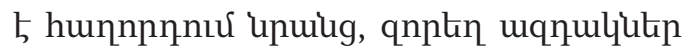
unuцhu puiumlymzhunıрjuiu qnpopupugh

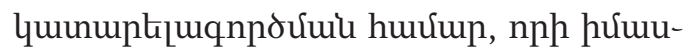
unnl buwnun umujumiutpnnu qhiulnnuquiuutiph ulupuiuputinn sutiunp 5 qhetiu

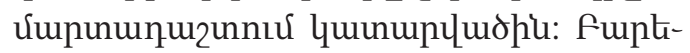

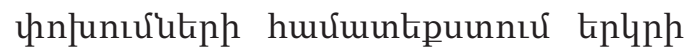

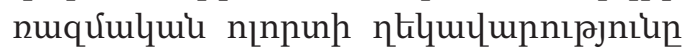
2 unniumblins 5 qunqughts umuntinuq-

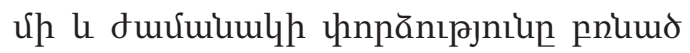

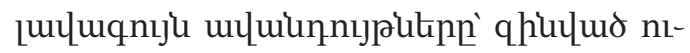

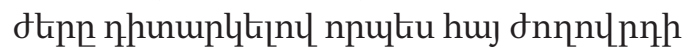
4Juiph, ukinnıрjuil punquцшбर्umu nı uknniunutiphu hqnp hujntiupp dunwuqtint tipu2fumunn [8]:

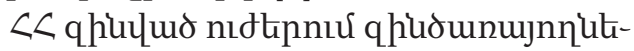

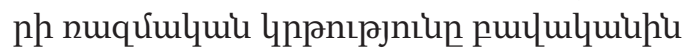

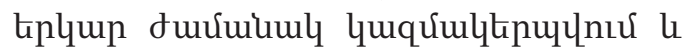
hpulquiumgunnu 5 p pupughl hpululyuiu

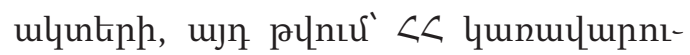

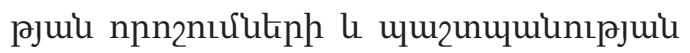

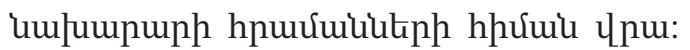
¿unhuiunıp hujtgulqunqujhi únunkg-

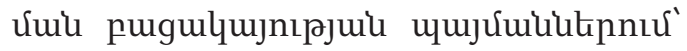

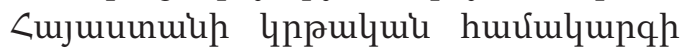
punlumgnıgh ưun luquínn nuqưulppulquil hưumlqunqn qunquintu $5 p$ uluq unkưuknnl, hurn ndцupnıpjniuukp 5 unuequgunsu qhuluo nıdtiph

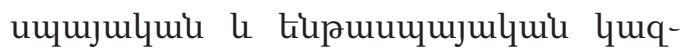
utiph qunpuujhi Glyntu punupulquilntpjuiu, upuiug npulyuцnnưuiu punăpug-

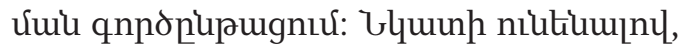

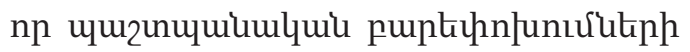

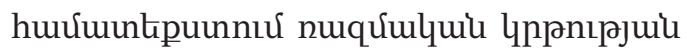
hưumlumqh qunqugntúp qtipulqu jut-

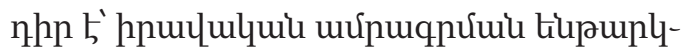
lthghu úhuuiumlyui hujkgulqupqujhi

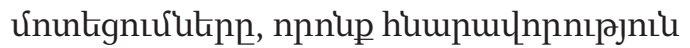

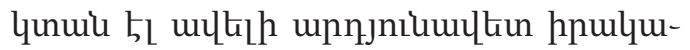
umgutıl pupān npulyuцnnnıu niukgnn

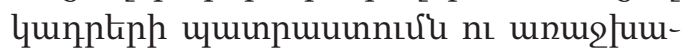
пugntún:

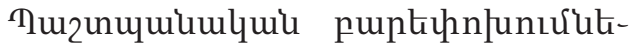
nh 2nquiumlnnu 2010 plulquih úmunhu

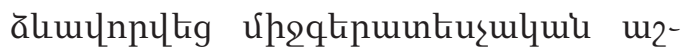
jumunuipujhi lunıup, nph uumunuly

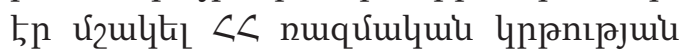
hujkgulqunqh umumqhon: foúph luwqunıu punqnlylt thu ukplumugnighsitip

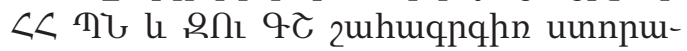

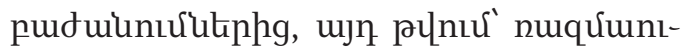

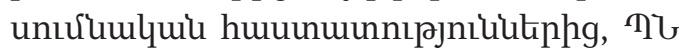
uqqujhi nuqưulupulyuu htinuqnunnıpjniuutiph hiuuunonnıung, hų̧uku

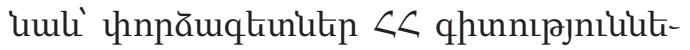
nh uqqujhu ulqunturumugh, $<<$ ynpntpjuiu li qhunnıрjuiu uurumpupnıpjniuhg, Eplumin ukinulymu huर्umumpuing,

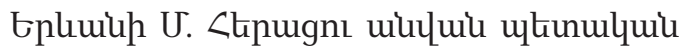

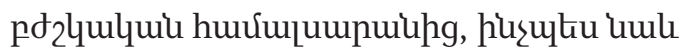

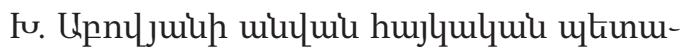




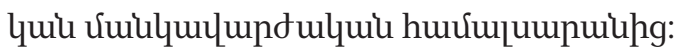

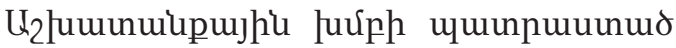

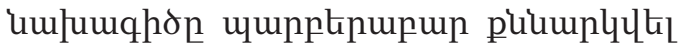

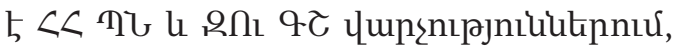
hul 2011 pumlumip pupugpntर' 'uul <u-

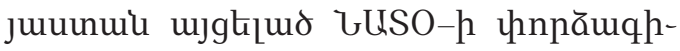
unulyuiu luर्uptiph htiun: Unn

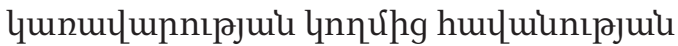
upduiumguil l. huunuunltight thptp

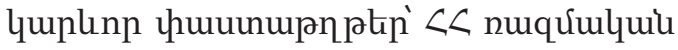

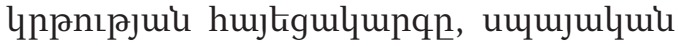

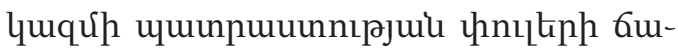

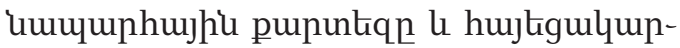

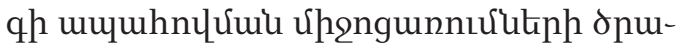
qhpn [9]:

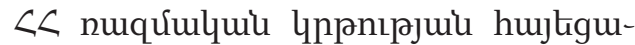
quinqny uwhưululnıर 5 hujtegulyunqh

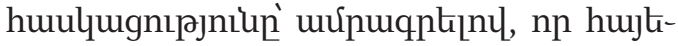

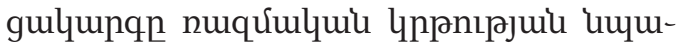

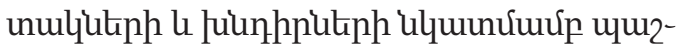

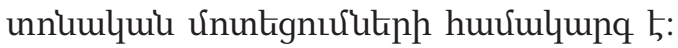

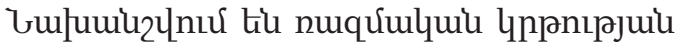
qnponn huruulumph puptuhn|untưtuph

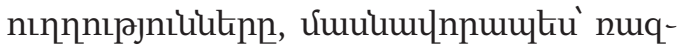

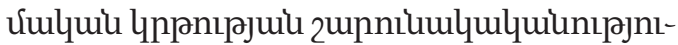
up, tinuump6uiu lppulquil huviulyupqh

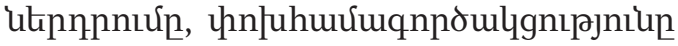
uqqujhi le uhguqqujhì ntuntúuulyuil huuunuunnipjnilutiph htin (hišutu qhiunpulquil, ujluute 5 l puinupughulquil), qhunulyuiu ns qhunuunthulplyuluiu qui-

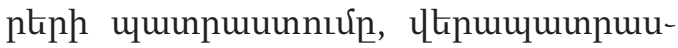

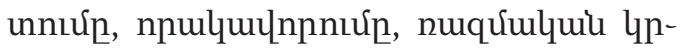
pnıрjuil huर्umlumqniर्u tiupuuuujulyuit

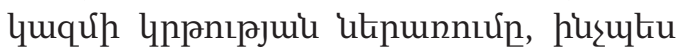

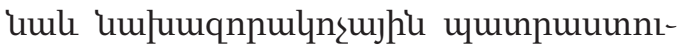
pjuiu nhunnıर्u' npultu uщujulquil uxum-

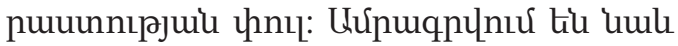

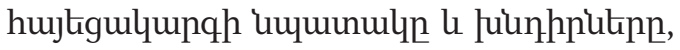
ujn punıu'

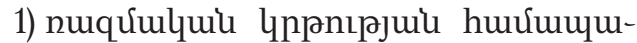
unuu|umiutgnıर्up utinulquil цppu-

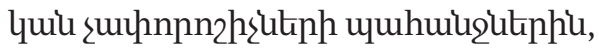

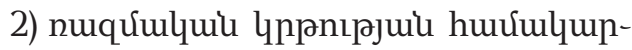
qh qunqugưuil le quinuptejuqnpo-

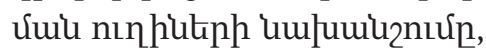

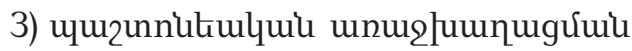
nınkqdp umlumiznứ,

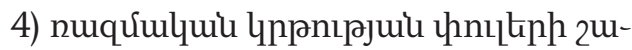

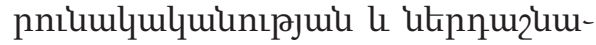

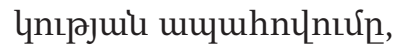

5) nuquulpppulyuil qnpopipugh lu-

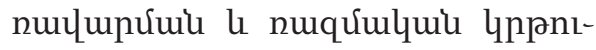

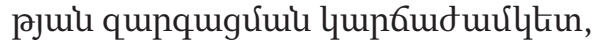
úpqumduर्ultin le tplyupudurulthen

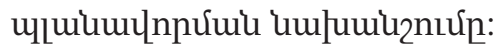

<ujtigulyunqnuर humultigunıu

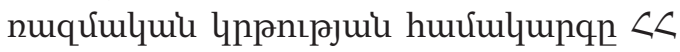
punhwiunıp lppulquil hưưlqupqh pun-

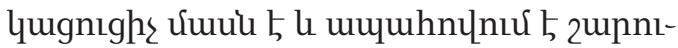

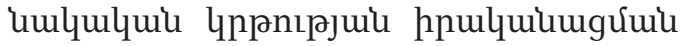
hưưp uihpudtzen lppulquil uhquqqujhi hưưqqnpoulgnıpjnil' jujunptil

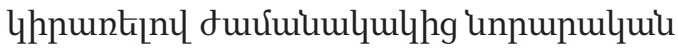

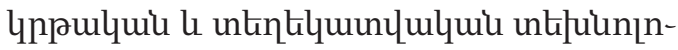

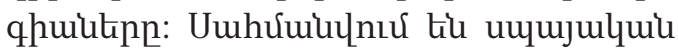

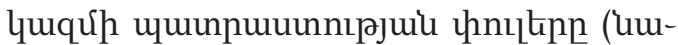

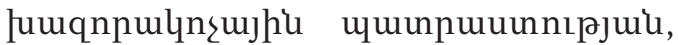
lpunutep, uцuq le pupăpuqnıju uщu-

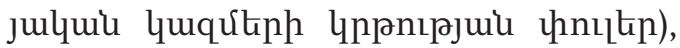
husute umb tipaumumulyul quqúp

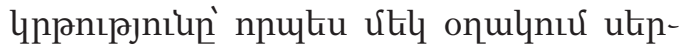

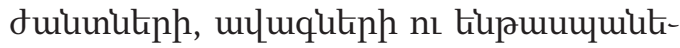

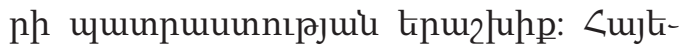

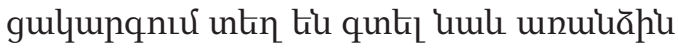
пpnujpluk, npnip ythpuptennuर tiu qhunulyuil le numulunumlyuil wiăhumlyuq-

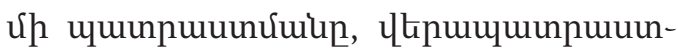
রulul nı npulyuцnnরưlu pupāpugưuin,

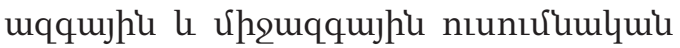
huuunuunıpjnitiutpp hưưqnpoul-

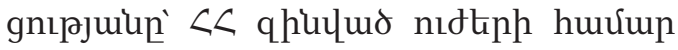

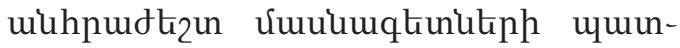
puuunर्umu, ounupteplpju utinnıрjuil qhunulppulyulu utipnıdp oqunuqnpoर्umu,

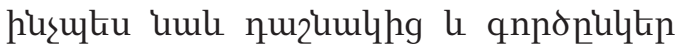


utinnıpjniuitiph qhulud nodteph htiu

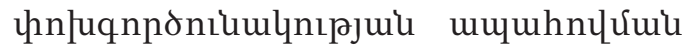

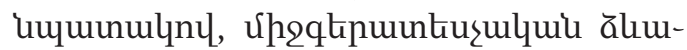

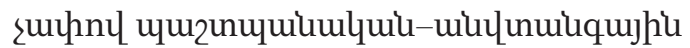
punupuqhunulquiu pupănuqnıju, htin-

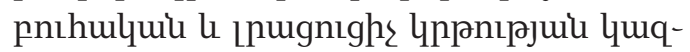
umlitpurumin:

Ujnuphunl hujtigulyunqny umpumil2ltighi nuquiulquil lnpnıpjuil qnnonn

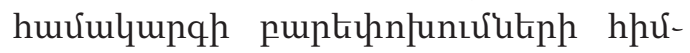

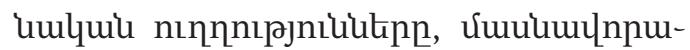

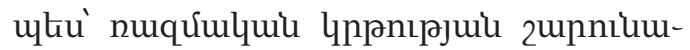

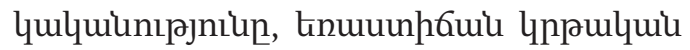
huर्umlumqh ukpnnnıर्u, hnjuhuर्umqnnowlgnıpjniup, qhunulquil le qhonu-

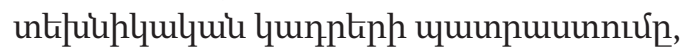

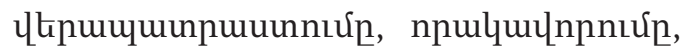

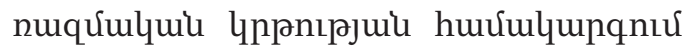

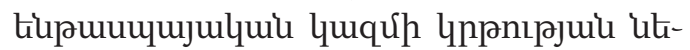

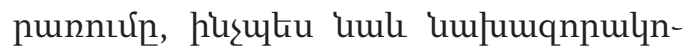

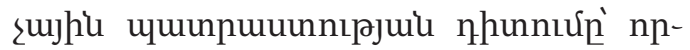

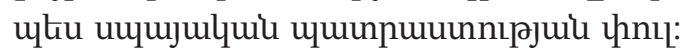

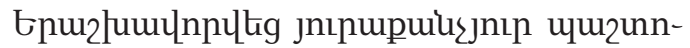

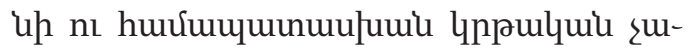

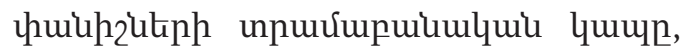
pupannugltig hujuunuiujuil punupughulquiu punăpuqnuju nıunıর'umlyui

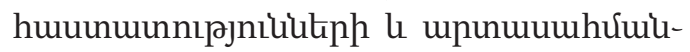
juiu nıunıর'umlumu huuunumnıpjniuutiph

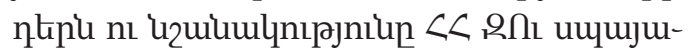

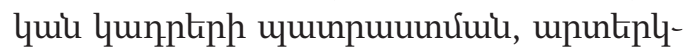

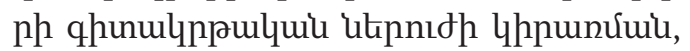

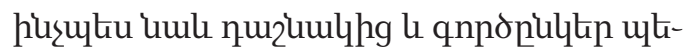
unnıرniuukph qhuluo nıdtiph hkiu thnju-

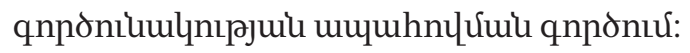

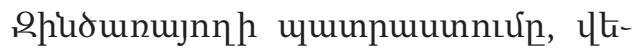

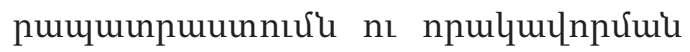

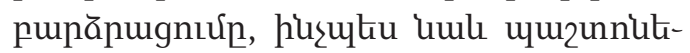
ulquu unwelumingnnún num qpunkg-

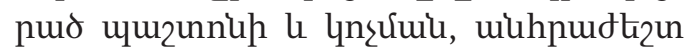

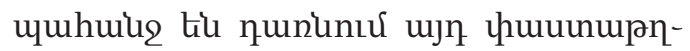

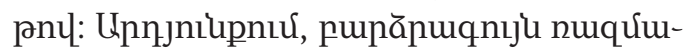

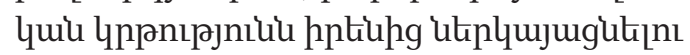

5 ns pte util pujlhg punlqugur lnpuquiu qnnoniutinıpjniu, ujL hưưuqunq,

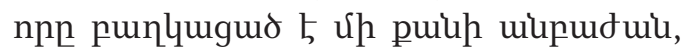

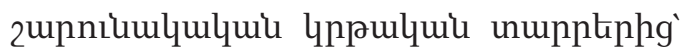

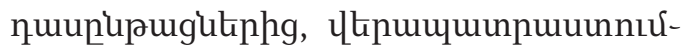

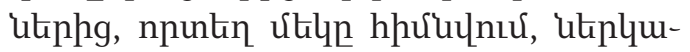

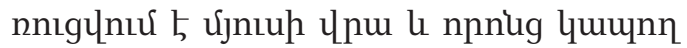
onulqu tiu huinhumiument qhudunujnnutıph dunujnıрjuiu nupugpnư ătinpptip-

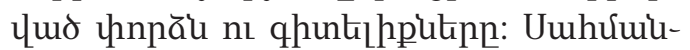
ltighu uml qhiumo nıdtiph uumumlumi

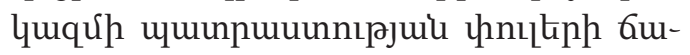
umumphujph pupntiqn ls nuqưulquil

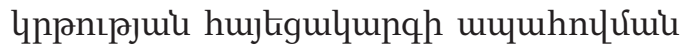
ungngunnứutinh ஓnuqhnn: Anutu uling-

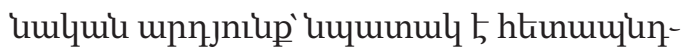

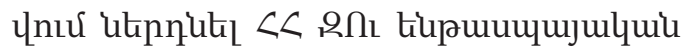

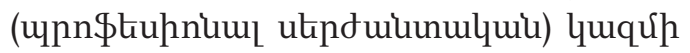

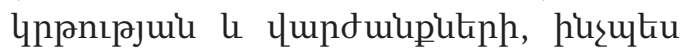

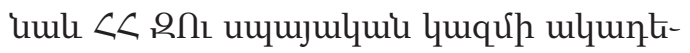

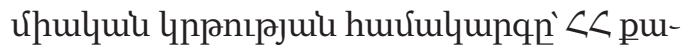

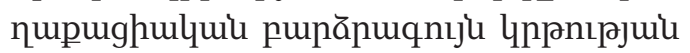

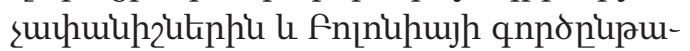
ghu hưưhnius:

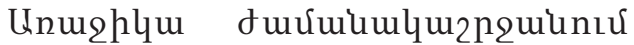
ulinp 5 niktiuml qhulnnulquil qunpte-

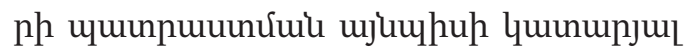

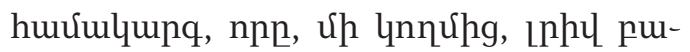
цunwph qhuцuo nıdtiph umhuiqum-

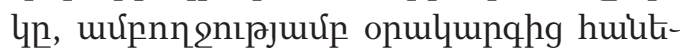

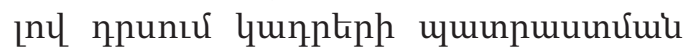
uuhpudtiznnıpjniun (hhuplt, lunupp sh quntu unuiuăhi uten úmulumqhunnt-

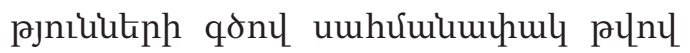

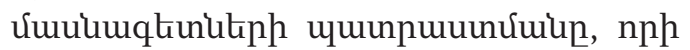
hnulquiumgnıúp untinnuर unuutiumuku

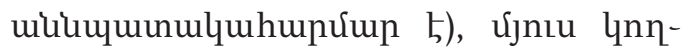
úhg, hp npulqulquiu pupăp রulqupnu-

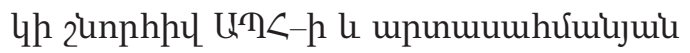
nnn2 kplputiphg <ujuuunuinıu lumpntp

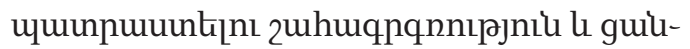

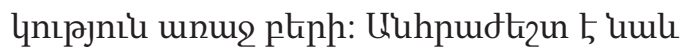

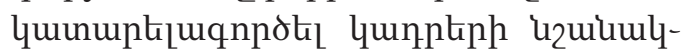




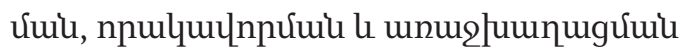
huर्umlumqn, nnukuqh puitulujht úp-

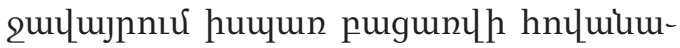

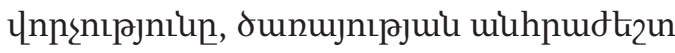

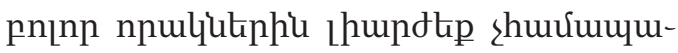

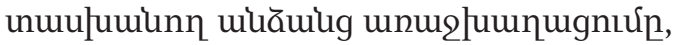

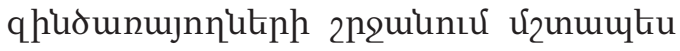

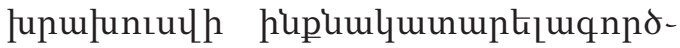
umu, úmulumqhumuluiu npwlyuцnnर्umu punănugưuù ăqunnứn: ?pu hưưun

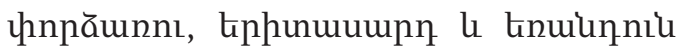
qhulnnulquil veuumqtinutiph uknnuzuml qunqugnıú' huj qhulnnu-

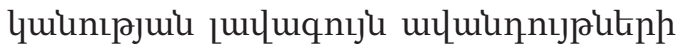

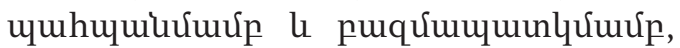

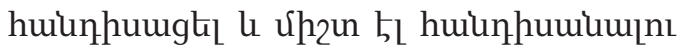

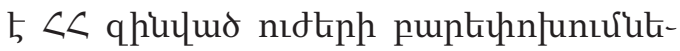
nh uihnudti2u nıntllhgn, puiuqh hujng

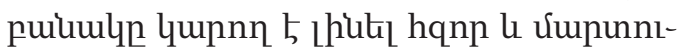
uml úpuju lthnghuhu quqúnıu niktium-

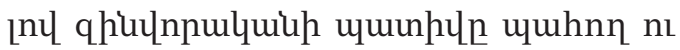

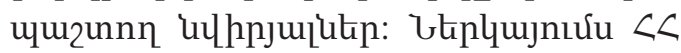

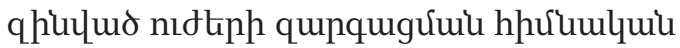

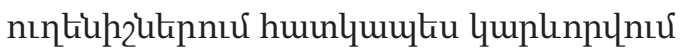
5 qhulnnwlquinıpjuiu दnpulquiu nı punnjuhnqtipuiumlquiu npwlutph pupkןu-

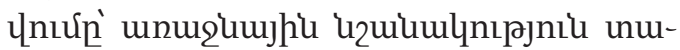
jnц uju qhudunujnnh lkpumphi, nl

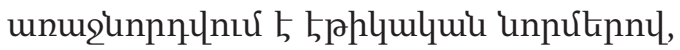
thundtipnptil oqunuqnnonuर्u 5 hptiu unp-

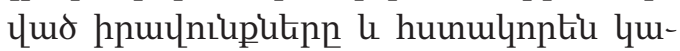

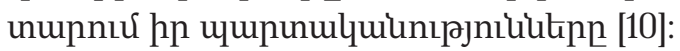

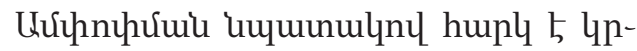

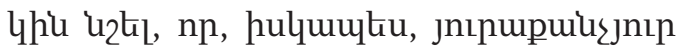

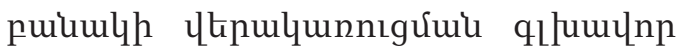
opjtilinu no unıpjtilinn umpuliunue qhudunujnnitin tiù npuku qhiuluo nıdt-

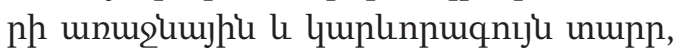

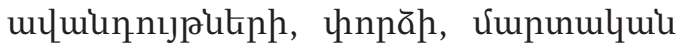
qupnnnıрjniulutiph ns punnjulquil nqnı

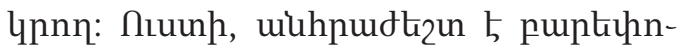

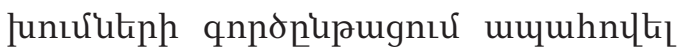

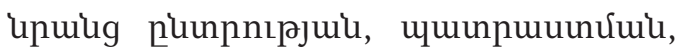

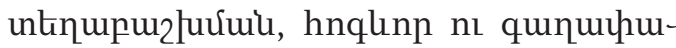

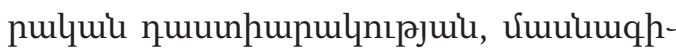

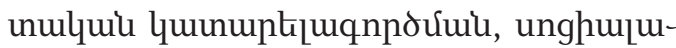

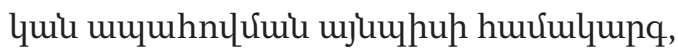
nph úpengny humpulnn thü ăluwunnts unp npulh huj qhiunnulumihu' hujptuhphi wuuwhưuiu ulhplud, punhuiunıр lu nuquauluiu junp qhuntilpputipnl nı pupăn lnıınnınujnц, hupunınnıju npn-

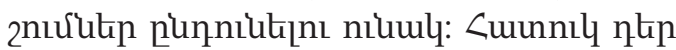

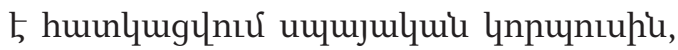

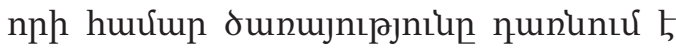
ljuilph nıntlihg, ltikumltinu, huumpu-

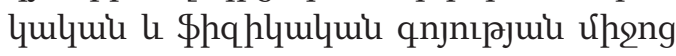
nı uxumumul:

funupughu ukinp 5 ăqunh qhuou-

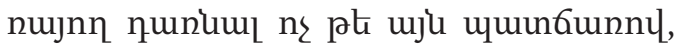
nn zh quinus hp untnn punupughu-

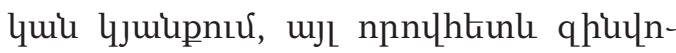
pulquiu dunujnıpjnilu umunцuptep $5 \mathrm{l}$

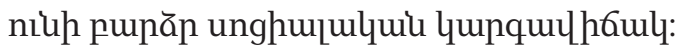
Uhuduruiuml puiumln ulkinp 5 himunu-

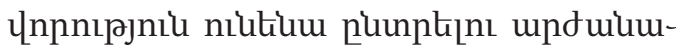
цnphi, juцuqnujupu, punniumlyhu lu ns pti qhulud nıdtiph zunptinn hưuminth unwehi umunuhwonl: Kling ujn hul ulquonunnứutinnl bl ukinnıpjniup ukinp b ătnumulh pnjnn úhongutinn, npukuqh qhulnnulquil úmulumqhunnpjniun hưumplh qnulhs, umunцuptep

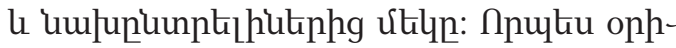

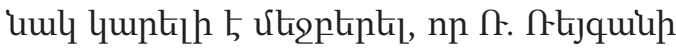

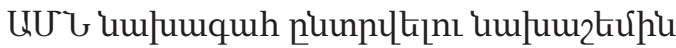

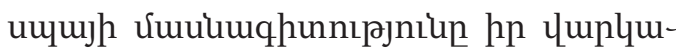

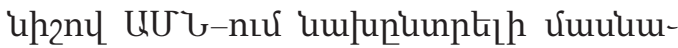

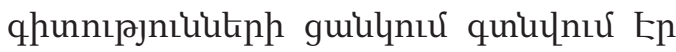
hhuqtennn unuulujulynuर, hul unw qu-

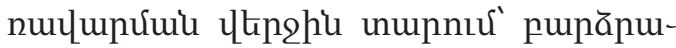
guul kрцnnnп untinn:

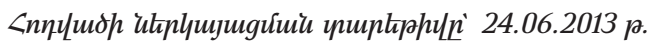




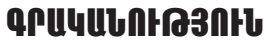

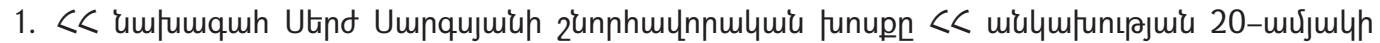

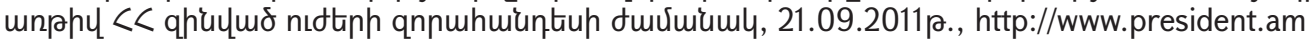

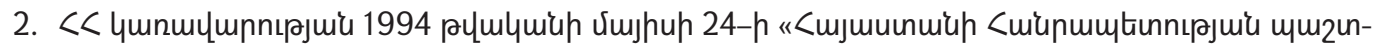

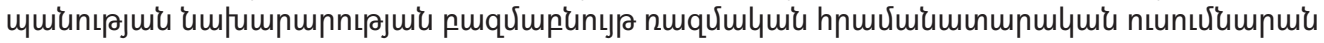
uuntindt_nı úmuhu» phy 279 nnn2nıu, http://www.arlis.am

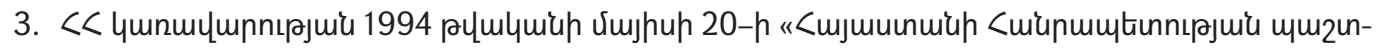

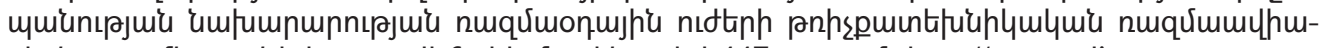

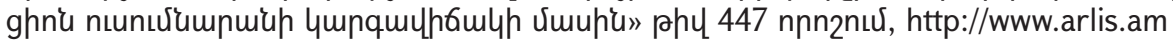

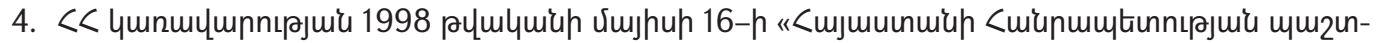

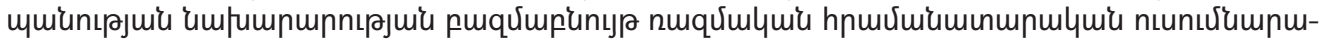

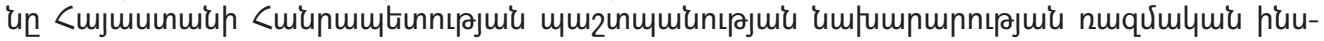

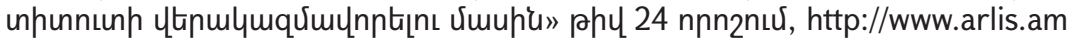

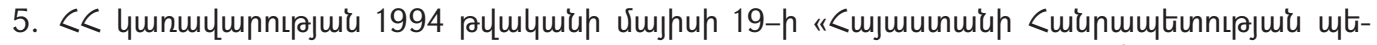

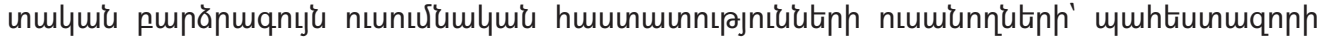

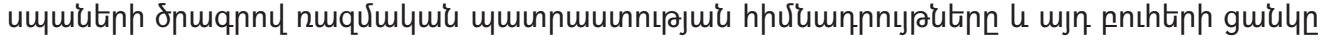
huuunuunt_nt Uuuup'u» phy 232 nnn2nıu, http://www.arlis.am

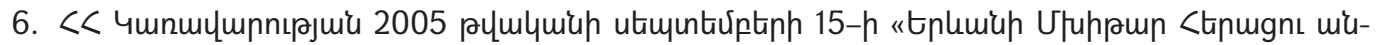

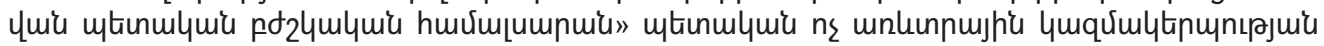

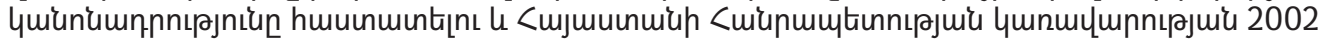

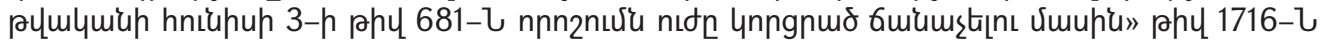
nnn2nux, <く TS 2005, hu. 71 (443), http://www.arlis.am

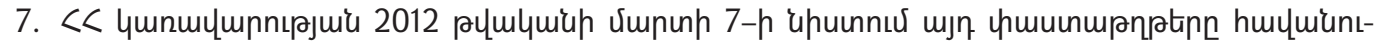

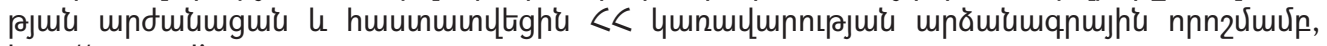
http://www.arlis.am

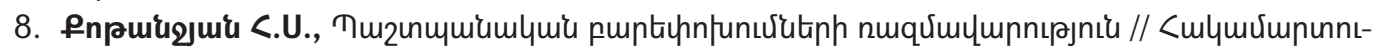

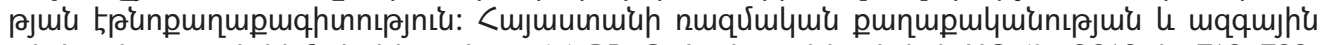

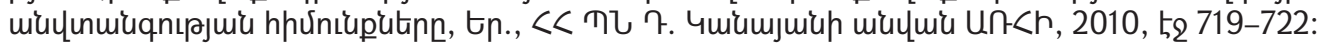

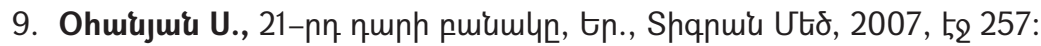

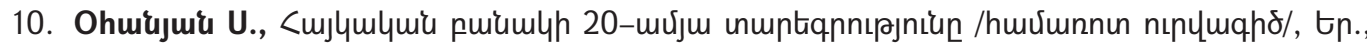
2012, t, 327

\section{ПОЛИТИКО-ПРАВОВОЕ КОНЦЕПТУАЛЬНОЕ РАЗВИТИЕ СИСТЕМЫ ВОЕННОГО ОБРАЗОВАНИЯ РЕСПУБЛИКИ АРМЕНИЯ}

\section{КОЧАРЯН ТИГРАН}

Начальник отдела юридического управления Министерство Оборонь РА, Советник 1-ого класса, доктор политических наук, доцент

В рамках формирования системного военного мышления с учетом своих национальных интересов важное место отводится модернизации военно-образовательной системы, повышению эффективности образовательных программ и учебных планов. Образование представляет собой процесс формирования фундаментальных, в том числе базовых специальных знаний, способности аналитически мыслить. Сфера военного образования и управления личным составом считается первичным фактором обеспечения функционирования оборонной системы и боеспособности ВС. 
В этом контексте рассмотрена история развития системы военного образования в ВС РА и анализируются основные направления ее развития. Реформирование системы военного образования осуществляется поэтапно по отдельным направлениям с расширением спектра изменений на основе достигнутых положительных результатов. Армянский офицер должен быть носителем и распространителем национальной идеологии. Одновременно он должен восприниматься общественностью как символ честности и образованности.

Дата представления статьи: 24.06.2013 г.

\section{POLITICAL AND LEGAL CONCEPTUAL DEVELOPMENTS OF THE MILITARY EDUCA- TION SYSTEM OF THE REPUBLIC OF ARMENIA}

\section{TIGRAN KOCHARYAN}

Head of the Branch of the Law Department of the Ministry of Defence of RA, 1-st Class Adviser,

Doctor of Political Sciences, Associate Professor

Within the framework of shaping systemic military thinking with regard for its national interests an important part is assigned to the modernization of the military education system, the increase of the effectiveness of educational programs and curricula. The education is process of forming fundamental, including basic special knowledge, ability to think analytically. The field of military education and personnel management is regarded as the primary factor for ensuring the defense system and combat capability of the Armed Forces.

In this context, the history of development of the military education system in the RA Armed Forces is examined, and the main directions of its development are analyzed. The reforming of the system of military education is gradually under way in separate areas by broadening the range of changes based on the positive results. The Armenian officer should be the bearer of the national ideology. At the same time, he must be perceived by the public as the symbol of honesty and education. 